\title{
El celular: dispositivo tecnológico para el desarro- llo de las competencias comunicativas
}

\section{The mobile phone: technological device for the development of communicative competences}

Fecha de recepción: 3 de Abril de 2016.

Fecha de aprobación: 27 de Noviembre de 2016.

Artículo de Reflexión
Diana Carolina Pineda Diaz* Ximena Gisele Rivera Guío** Sandra Milena Murcia Pardo***

\section{Resumen}

Este artículo de reflexión se deriva de la investigación "Competencia comunicativa oral por medio del uso del celular", cuyo objetivo fue identificar cómo el uso del dispositivo móvil incide en la mejora de la competencia comunicativa oral de los estudiantes de grado noveno en la Institución Educativa Técnica de Nobsa, a través del análisis e interpretación sobre la manera como la tecnología ha influido en la calidad de la educación, sus aportes a la misma y los avances que se han obtenido en el desarrollo de habilidades cognitivas y competencias comunicativas. A partir de un sustento teórico, se busca delimitar los conceptos que dan soporte al proceso investigativo que posee un enfoque crítico social generando un cambio de pensamiento en el contexto educativo. Así mismo, el trabajo se enmarca en la Investigación Acción Educativa. En consecuencia, se presentan los impactos alcanzados con una propuesta que usó el celular como herramienta pedagógica, en talleres
*Institución Educativa Técnica de Nobsa- Boyacá Colombia dayan1722@hotmail.com

**Institución Educativa Técnica de Nobsa, Boyacá Colombia

ximonarivera@hotmail.com ***:Universidad Pedagógica y Tecnológica de Colombia, Boyacá - Colombia sandra.murcia@uptc.edu.co

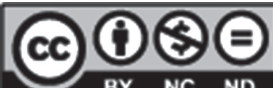


que lograron potenciar algunas de las habilidades necesarias para alcanzar una competencia comunicativa oral adecuada, y cómo se creó un ambiente en donde los estudiantes con motivación, trabajo colaborativo e innovación aprovecharon las características pedagógicas que puede brindar el

\section{Abstract}

This article of reflection derives from the research "oral communicative competence through the use of cell phones", whose objective was to identify how the use of the mobile device affects the improvement of the oral communicative competence of ninth grade students in the Educational Institution of Nobsa (Colombia), through the analysis and interpretation of how technology has influenced the quality of education, its contributions to it and the advances that have been made in the development of cognitive skills and communication skills. From a theoretical basis, it seeks to define the concepts that support the research process that has a critical social approach generating a change of thought in dispositivo al ser multifuncional, asequible y portátil.

Palabras clave: teléfono móvil, tecnología de la información, expresión oral, educación, innovación pedagógica, motivación. the educational context. Likewise, the work is part of the Educational Research-Action. Consequently, the impacts achieved are presented with a proposal that used the cell phone as a pedagogical tool, in workshops that managed to enhance some of the skills needed to achieve an adequate oral communication competence, and how an environment was created where students with motivation, Collaborative work and innovation took advantage of the pedagogical features that the device can offer by being multifunctional, affordable and portable.

Keywords: mobile phone, information technology, oral expression, education, pedagogical innovation, motivation. 


\section{Introducción}

El presente escrito es una reflexión fruto del proceso de investigación intitulado "Competencia comunicativa oral por medio del uso del celular", preocupado por favorecer la calidad de la educación, la motivación en los estudiantes, la innovación en la práctica pedagógica y mejorar la competencia comunicativa oral, a partir del uso de la herramienta tecnológica con mayor popularidad y aceptación entre las nuevas generaciones: el celular.

Partiendo de la premisa de que los jóvenes del siglo XXI hacen parte de una generación de nativos digitales que piensa y aprende de forma interactiva, $y$ se mueven en un ambiente virtual que atrapa su atención dejando de lado sus obligaciones académicas más prioritarias; se considera oportuno introducir la tecnología al ambiente de aprendizaje, como una herramienta didáctica y potenciadora en el desarrollo de las diversas competencias.

Una de las herramientas o dispositivos tecnológicos de mayor acogida en la actualidad, es el teléfono móvil (celular), el cual -por tendencia o por necesidadse ha convertido en un protagonista de los bienes de muchas personas. En el entorno educativo, a este dispositivo se le ha tildado de «distractor» e impedimento en el proceso de aprendizaje y enseñanza, pasando por alto la diversidad de usos, ventajas y oportunidades que se derivan de su aplicación en el contexto del aula.

La Institución Educativa Técnica de Nobsa no es ajena a esta realidad. El uso del teléfono móvil se encuentra prohibido dentro de la misma, debido al manejo no adecuado que los estudiantes hacen de él; su empleo se limita a la comunicación con sus pares, traspaso de información e intereses personales, como música y juegos.

Por otro lado, se presenta una escasa participación oral en las diferentes clases, reflejada en el nerviosismo, poca seguridad al expresar sus ideas, uso inapropiado del lenguaje (vulgaridad); producto, tal vez, de la falta de instrucción para hacerlo correctamente y a la poca relevancia que se le ha dado a la habilidad comunicativa oral.

Con la intención de renovar la visión que se tiene de este dispositivo, la investigación de las autoras pretendió determinar cómo el uso del dispositivo incide en el mejoramiento de la competencia comunicativa oral expositiva de los estudiantes, a través de la creación y de la aplicación de una estrategia pedagógica.

Después de indagar lo que hasta este punto se ha estudiado sobre la incursión del dispositivo en la educación, y su uso con fines pedagógicos para desarrollar algún tipo de habilidades, se evidencia la poca existencia de investigaciones que lo tomen como medio de innovación pedagógica. A cambio, se halló una gran cantidad de estudios orientados hacia desarrollar o mejorar habilidades cognitivas o competencias comunicativas por medio del uso de herramientas tecnológicas o de información; estas, por ser las más cercanas al proceso que se lleva a cabo, se tomaron como el soporte que le da la base epistemológica al marco teórico de la investigación.
El presente escrito es una reflexión fruto del proceso de investigación intitulado "Competencia comunicativa oral por medio del uso del celular", preocupado por favorecer la calidad de la educación, la motivación en los estudiantes 
A partir de la reflexión en el desarrollo del proceso de esta investigación-acción educativa, se dio evidencia de que se comparten las apreciaciones del referencial teórico citado
En este sentido, es iniciativa de las investigadoras retomar esos componentes teóricos, los resultados, las conclusiones o los aportes, que han dado al campo educativo; y la manera (didáctica) como han utilizado las diferentes herramientas tecnológicas los docentes y profesionales de la educación en el aula, para que, a partir de un proceso investigativo con enfoque crítico social que se desarrolló, se origine un nuevo elemento o fuente de información que pueda generar otras posibles investigaciones.

A partir de la reflexión en el desarrollo del proceso de esta investigaciónacción educativa, se dio evidencia de que se comparten las apreciaciones del referencial teórico citado; igualmente, los contenidos analizados e incorporados aportan valiosos planteamientos para la creación de la propuesta pedagógica que se aplicó.

La propuesta creada tomó como herramienta principal el teléfono móvil usado durante la aplicación de una prueba diagnóstica, tres talleres y una prueba final, cada uno de los anteriores evaluados con una rúbrica que permitieron identificar las debilidades persistentes y los progresos alcanzados durante su desarrollo.

Los resultados obtenidos con los estudiantes de grado noveno de la Institución Educativa Técnica de Nobsa, mostraron la importancia que poseen la expresión oral y los saberes previos en el mejoramiento del desempeño académico de los estudiantes, el perfeccionamiento de algunas habilidades necesarias para la competencia comunicativa oral, como el autodominio, los aspectos propios del paralenguaje (dicción, tono, fluidez, ritmo y entonación), y la kinestesia (postura, gestos, contacto visual, expresión facial y ademanes). Además, se evidenció el aumento de la motivación y el gusto por participar en las diferentes actividades donde el dispositivo de comunicación móvil (celular) fue la herramienta innovadora.

\section{Antecedentes}

En la búsqueda de información sobre estudios realizados con el fin de desarrollar la competencia comunicativa oral (o que mencionasen el celular como herramienta pedagógica), se encontró una serie de investigaciones encaminadas al uso de las Tecnologías de la Información y las Comunicaciones (TIC) en los procesos de aprendizaje y enseñanza, o para el mejoramiento o el desarrollo de habilidades cognitivas y competencias comunicativas. De estas investigaciones, se tomaron los resultados, y se estableció la influencia de la tecnología en el desarrollo de las diferentes competencias.

De manera complementaria, se hace referencia a algunos artículos que, específicamente, abordan la oralidad, lo que esta representa, sus implicaciones en el ámbito educativo y las conclusiones a las que han llegado las investigaciones analizadas. Se han clasificado los estudios según las siguientes categorías, con el fin de encontrar puntos en común para su análisis y discusión.

Una de las categorías está determinada por las investigaciones que tienen como objetivo el desarrollo de la competencia 
comunicativa del inglés como lengua extranjera, que centraron su interés en aplicar estrategias a partir del uso de diferentes tecnologías sincrónicas y asincrónicas que buscaban desarrollar o fortalecer la expresión oral de los estudiantes objeto de estudio. Aunque tales estudios no comparten semejanzas totales en cuanto al idioma o tipo de investigación, demostraron en sus resultados que un gran porcentaje de los estudiantes, fue beneficiado con la aplicación de actividades donde se usaban dispositivos o medios tecnológicos (internet, ambientes virtuales de aprendizaje, podcasts ${ }^{1}$, videoconferencias, chats, entre otros), comparados en algunos casos, con grupos en los que no se habían usado.

El desarrollo de la expresión oral en los estudiantes de inglés, resalta la relevancia de la comunicación no verbal, de la motivación $\mathrm{y}$, principalmente, de la necesidad de aplicar estrategias en forma gradual que no obliguen a aquellos estudiantes que, en un principio, no quieren participar de las actividades, generándoles confianza y un ambiente de cooperación y reflexión constante. La aplicación de estrategias motivantes genera la participación en clase y, a su vez, la reflexión y la creación de conciencia sobre su aprendizaje, debilidades, gustos y posibles soluciones (Narváez, 2014).

A través de esa reflexión y creación de conciencia, se logra incrementar la autonomía en el estudiante, facultad que debe estar presente en el proceso de aprendizaje, en la toma de decisiones y en su autoevaluación; influyendo a largo plazo en el desarrollo de algunas de las habilidades más importantes de la competencia comunicativa oral, como lo son el autodominio y la kinestesia.

Otra estrategia adecuada para el desarrollo de la expresión oral en la lengua extranjera, es el uso de podcast. Estos archivos son empleados como modelos de comunicación o para revisar la producción oral, permitiendo su reproducción las veces que sean necesarias. Para su creación, es necesaria la participación de más de dos personas, en la mayoría de los casos, razón por la cual se fomenta el trabajo colaborativo. Durante este estudio, se lograron obtener materiales llamativos e innovadores que permiten asociar la educación con la diversión.

Las anteriores conclusiones son confirmadas en el artículo "Los podcast como herramienta de enseñanzaaprendizaje en la universidad" de autoría de Ramos y Caurcel quienes los utilizaron como propuesta didáctica encaminada a introducir las TIC, el trabajo autónomo y la tarea colaborativa. Se llegó a la conclusión de que, por medio de esta herramienta, se potenciaron los diferentes tipos de aprendizaje y la competencia tecnológica (Ramos y Caurcel, 2011).

Más allá de los alcances de esta investigación, se puede inferir que los podcast son un medio muy motivador que genera interés en los estudiantes a través de su ejercicio comunicativo, produce trabajo en equipo, lo cual permite valorar su propio desempeño y la aceptación que entregan los demás, y el fortalecimiento de los niveles de confianza en el educando.

En el artículo "Desarrollo de la competencia comunicativa en el idioma
${ }^{1}$ Publicación digital periódica en audio o video, creada o descargada de internet. 
En síntesis, una de las similitudes en los resultados de estas investigaciones, fue el aumento de la autonomía de los estudiantes, ya que la flexibilidad dada en la realización de las diversas tareas o actividades, proporcionaba una oportunidad de toma de decisiones y libertad al escoger las estrategias para realizar los trabajos. inglés en una plataforma interactiva”, se presentan los resultados de una investigación llevada a cabo en el centro de capacitación superior para el trabajo industrial (Cecati) en México, en la que hicieron uso de una plataforma interactiva en línea para el aprendizaje del inglés. En esta investigación cualitativa, no se evidenció el resultado esperado debido a la falta de autodisciplina y compromiso, tanto de estudiantes como de docentes.

La investigación tenía unos propósitos establecidos en procura de mejorar el nivel de suficiencia en la segunda lengua; pero, debido a la falta de compromiso y poca responsabilidad mostradas por los participantes en esta estrategia, no se lograron los resultados esperados (Rosales \& Zarate, 2013). Estas debilidades revelan uno de los riesgos a los que se enfrenta el desarrollo de investigaciones en las que se usan las TIC, pues si falta realimentación, planeamiento y acompañamiento, los objetivos no se pueden llevar a buen término.

A su vez, demuestra la importancia que, por un lado, el docente debe darle a la planeación estratégica de sus clases y del uso de tecnologías de la comunicación en ellas; y por el otro, al compromiso, motivación y autonomía que se debe despertar en los estudiantes al hacerlos partícipes de su autoevaluación.

En consecuencia, se pueden identificar algunas debilidades que posee el uso de las tecnologías sincrónicas, ya que los estudiantes no son ajenos a problemas de conectividad, incompatibilidad en características técnicas o a la falta de conocimiento del manejo de las herramientas que pueden dificultar los procesos o actividades de cualquier trabajo investigativo.

En síntesis, una de las similitudes en los resultados de estas investigaciones, fue el aumento de la autonomía de los estudiantes, ya que la flexibilidad dada en la realización de las diversas tareas o actividades, proporcionaba una oportunidad de toma de decisiones y libertad al escoger las estrategias para realizar los trabajos. Así mismo, el aumento en la motivación fue un punto en común encontrado en ellos. El uso de las tecnologías para asociar la diversión con la educación, permite mejorar la calidad del proceso educativo y la superación de barreras de espacio y tiempo. También, demuestra que al estimular la motivación se mejora el aprendizaje, pues el estudiante toma un rol activo en su desarrollo cognitivo.

Del mismo modo, se encuentra una categoría conformada por las investigaciones que buscan desarrollar la competencia comunicativa tomando el español como segunda lengua. Estas investigaciones delimitaron su campo de acción en grupos de estudiantes interesados en adquirir o mejorar su suficiencia en el idioma español.

En el artículo "Mis estudiantes son «diginativos», ¿y los tuyos? El aprendizaje de la competencia comunicativa oral a través de entornos digitales", se plantea un reto posible como lo es superar el rechazo hacia las actividades académicas y acercarse al perfil digital de los alumnos, explorando las posibilidades que ofrecen las nuevas tecnologías para enriquecer las clases. 
En los últimos años, el material audiovisual que se encuentra en la internet ha aumentado y ayuda significativamente a trabajar y/o desarrollar aspectos interculturales, quinésicos y proxémicos de la comunicación, con gran variedad de géneros: noticias, entrevistas, avances de películas de cine, reportajes culturales, anuncios, videos y documentales creados con fines educativos, explotaciones didácticas, e incluso grabaciones privadas (Jáuregi y Sanz, 2006). Algunos de estos recursos pueden ser utilizados en la investigación para ejemplificar la forma correcta de expresión oral o como producto de las actividades.

Los autores, de esta manera, dan a conocer, implícitamente, la realidad de la generación actual con la que se cuenta como docentes, al reconocer que ella evoluciona con la tecnología, dominando de forma natural las herramientas y el lenguaje digital; y además la innumerable cantidad de recursos tecnológicos que se pueden aprovechar en pro de mejorar la educación a través de la innovación.

Lo anterior se confirma en la tesis de doctorado titulada "El desarrollo de la competencia oral en la enseñanzaaprendizaje del español como lengua extranjera a través del uso de las tecnologías de la información y la comunicación", donde se plantea la importancia de incorporar dichas tecnologías a los procesos pedagógicos y de evaluación en las instituciones educativas, con el propósito de colocar al sistema educativo a la vanguardia de la sociedad del siglo XXI.

Su objetivo se centra en el uso de las TIC para el desarrollo de la competencia discursiva oral, en la enseñanzaaprendizaje del español como lengua extranjera; aunque la mayoría de estos recursos no haya tenido en su origen una intención didáctica, se genera un modelo que sirve para el diseño de programas de instrucción, de materiales didácticos, de aprovechamiento de recursos y de evaluación de la competencia oral. Trata de validar unos principios teóricos sobre el desarrollo de la lengua oral y principios pedagógicos relacionando la teoría con la práctica.

La investigación afirma cómo las herramientas tecnológicas pueden ser positivas en el ámbito educativo, adquiriendo un papel didáctico en el perfeccionamiento de la competencia comunicativa oral de los estudiantes; por ejemplo, permitiendo almacenar voz, video o imagen de manera comunicativa, promoviendo la ética en su uso (Martínez, 2012).

Desde otro punto de vista (y de acuerdo con el contexto en el que están sumergidos los estudiantes en la actualidad), el uso del internet se ha convertido en un medio clave para desarrollar competencias cognitivas y destrezas personales como la autonomía. Estas premisas son confirmadas en el artículo "El desarrollo de la metacognición y de la competencia estratégica oral mediante el uso de internet”. En él, se expresa cómo la comunicación no debe manejarse solo durante la interacción, sino también antes y después de estar resolviendo problemas lingüísticos que se pueden presentar. Lo anterior se logra a partir de la inclusión del docente en el proceso, no solo de enseñar e impartir conocimientos, sino también en el de
Los autores, de esta manera, dan a conocer, implícitamente, la realidad de la generación actual con la que se cuenta como docentes, al reconocer que ella evoluciona con la tecnología, dominando de forma natural las herramientas y el lenguaje digital 


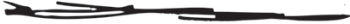

Por último, los trabajos investigativos interdisciplinares enmarcados en el mejoramiento de competencias a través de las TIC, proponen mejorar las competencias comunicativas desde diferentes áreas y niveles de la educación haciendo uso también de las TIC. crear -de manera óptima- estrategias, y planear clases (Ospina, 2011).

Igualmente, esta red de comunicación permite acceder a una inmensa cantidad de información, en donde la oferta en línea de páginas con videos educativos, páginas interactivas, software acoplable a las clases o plataformas de comunicación e interacción, se convierten en una herramienta amigable que contribuye a la labor docente, condicionada por un uso estructurado y adecuado para el logro de los objetivos propuestos.

En cuanto a las investigaciones que desarrollan las habilidades comunicativas en un entorno donde la lengua española es la extranjera, se encuentran tres aspectos afines:

El primero de ellos es la necesidad del docente de acercarse al perfil digital de los estudiantes, debido a su acelerada incursión en las nuevas tecnologías y en cada uno de los procesos que ellas conllevan. Este acercamiento debe centrarse en desafiar lo tradicional, pues el docente actualmente permanece ajeno -y más que ajeno «prevenido»- a las TIC, y es ignorante de los beneficios que ellas aportan a los procesos de aprendizaje y enseñanza.

En segundo lugar, se encuentra que el aspecto cultural de los protagonistas del proceso educativo, debe ser tomado en cuenta a la hora de planificar y evaluar las actividades, para así permitir una comunicación efectiva y significativa. Lo anterior, atendiendo a que un estudiante se podrá desempeñar mejor en un contexto conocido y acorde a sus intereses.
En tercer lugar, la mayoría de los estudios coinciden en darle importancia al trabajo colaborativo como medio de desarrollo de las tareas o actividades comunicativas. En el desempeño docente, el fomentar este tipo de análisis logra no solo crear autonomía, sino también -a partir de grupos pequeños heterogéneos- tratar de cumplir una meta en común que genere cooperación, integración y enseñanza más reflexiva, que además aplicarán los estudiantes en lo académico y en su desarrollo personal.

Por último, los trabajos investigativos interdisciplinares enmarcados en el mejoramiento de competencias a través de las TIC, proponen mejorar las competencias comunicativas desde diferentes áreas y niveles de la educación haciendo uso también de las TIC. Estas investigaciones coinciden en la importancia que tiene el rol del docente para la efectividad del aprendizaje y el desarrollo de diversas habilidades en los estudiantes. Esto quiere decir que no solo a través de los conocimientos que posee el docente se puede llegar a una calidad educativa, sino que es más importante la manera en la que él ejerza su labor, planeando cada una de las actividades y estrategias; si hace uso de las TIC, debe saber cuál es la apropiada para la tarea que pretende realizar.

Además, durante la aplicación de las metodologías de estas investigaciones, se logró -intencionalmente o no- la individualización de la enseñanza, pues se tenían en cuenta el contexto de los alumnos, las necesidades, los gustos, los intereses y los estilos de aprendizaje, enriqueciendo los procesos educativos $\mathrm{y}$ 
fomentando, a su vez, la autonomía y la participación activa de los estudiantes. Todo esto también trajo como beneficio la motivación en ellos, quienes mostraron un mayor interés por el desarrollo de las actividades $\mathrm{y}$, sobre todo, por su desempeño y autoevaluación.

El proyecto de aprendizaje móvil aplicado en una institución educativa privada en la ciudad de México, plantea la importancia del uso del M-learning como práctica funcional y flexible. Por medio del análisis de los recursos móviles de una muestra, se buscaba identificar cómo promover el desarrollo de habilidades cognitivas en los estudiantes. En cuanto a la metodología, usó entrevistas, encuestas, análisis de documentos y observación no intrusiva. Los resultados indicaron que el uso de recursos M-Learning modifica el ambiente de aprendizaje, al convertir cualquier escenario en un ambiente innovador y colaborativo (Ramos, 2010).

Se puede afirmar que, con el uso de los dispositivos móviles, se obtiene el conocimiento desde cualquier lugar -en cualquier momento y sin ataduras físicaslo cual provoca una revolución educativa, establecida de acuerdo con las necesidades de una sociedad cambiante. Además, son una herramienta indispensable en la actualidad para el proceso de aprendizaje y enseñanza; facilitan la construcción del conocimiento, la transmisión de ideas, y el desarrollo de destrezas, habilidades o competencias comunicativas.

De esa manera, las TIC ofrecen oportunidades para innovar y cualificar la enseñanza de las habilidades comunicativas en modelos educativos como el de 'Escuela Nueva' a través de diferentes estrategias. Se ha logrado incorporar las TIC en el desarrollo del currículo con una interacción dinámica y atractiva, fomentando la motivación, la autonomía y la libertad en el proceso de aprendizaje de los estudiantes (Monsalve, 2009).

Eldesarrollodehabilidadescomunicativas se puede realizar en niveles y modelos pedagógicos diferentes con el fin de buscar la calidad educativa, atendiendo a las características y necesidades de distintas poblaciones. Este proceso conlleva una interacción dinámica de los estudiantes y su inserción en la vida activa que -a largo plazo- genera trabajo colaborativo.

\section{Consideraciones teóricas que favorecen la reflexión del proceso investigativo}

A partir de investigaciones que aportan una aproximación a la construcción del proceso de la investigación que ha sido referido, las autoras han estructurado el proceso investigativo teniendo en cuenta ciertos componentes teóricos que aportaron significativamente de esta forma:

Primero, desde la competencia comunicativa, la cual se entiende como la capacidad que abarca tanto el conocimiento de la lengua como la habilidad para utilizarla (Hymes, 1996); es el término más general para la capacidad comunicativa de una persona. La adquisición de tal competencia está mediada por la experiencia social, las necesidades y motivaciones, y la acción, que es -a la vez- una fuente renovada de estímulos, exigencias y experiencias.
Se puede afirmar que, con el uso de los dispositivos móviles, se obtiene el conocimiento desde cualquier lugar -en cualquier momento y sin ataduras físicas- lo cual provoca una revolución educativa, establecida de acuerdo con las necesidades de una sociedad cambiante. 
Se puede afirmar entonces, que la competencia comunicativa oral es integral al involucrar actitudes, valores y motivaciones, relacionadas con la lengua, con sus características y sus usos, y con los demás sistemas de comunicación.
Segundo, solo existen cuatro formas distintas de uso de la lengua según el papel que desempeñen los individuos en el proceso comunicativo: hablar, escuchar, leer y escribir. Esos usos de la lengua se transforman en habilidades lingüísticas según el código oral o escrito, y el papel receptivo o productivo que tengan en la comunicación (Cassany \& Luna, 1998).

Para el caso específico de estainvestigación, el objeto de estudio es la competencia comunicativa oral, es decir aquella actividad de intercambio comunicativo que posee como código y que requiere el desarrollo de dos habilidades, destrezas, capacidades lingüísticas particulares: hablar y escuchar.

De este modo, el desarrollo de una competencia comunicativa implica conocer la oralidad, cuyo término es una expresión que se empezó a utilizar en los años 60, como resultado de investigaciones realizadas en diferentes disciplinas, entre ellas la antropología, la literatura y las nuevas tecnologías de la comunicación, las cuales han mostrado interés por abordar «lo oral» desde especificaciones diferentes, pero en su mayoría desde una perspectiva analítica. Sin embargo, y a pesar de ese creciente interés multidisciplinar por la oralidad, no existe un consenso epistemológico que aclare el difuso panorama sobre la naturaleza de este concepto (Abascal, 2002).

Los seres humanos adquieren y generan la capacidad de hablar o callar, y también sobre qué hacerlo, con quién hacerlo, dónde, para qué y en qué forma. Es decir, se adquiere un conocimiento no solo gramático de la lengua, sino de sus diferentes registros y su pertinencia, volviéndose capaces de interactuar en eventos comunicativos, evaluando su participación y la de los otros (Abascal, 2002).

Se puede afirmar entonces, que la competencia comunicativa oral es integral al involucrar actitudes, valores y motivaciones, relacionadas con la lengua, con sus características y sus usos, y con los demás sistemas de comunicación. La lengua no tiene como única función el nombrar, sino que también se organiza para lamentarse, alegrarse, rogar, prevenir, defender, atacar; relacionadas con las diferentes formas de persuasión, dirección, expresión y juegos simbólicos.

Como lo mencionan autores especializados en el discurso oral (Núñez, 2004), se conoce una clasificación según el modo del discurso usado: narración, descripción, exposición, argumentación, la cual se toma como referente para delimitar aún más la temática a competencia comunicativa oral expositiva.

\section{Tecnologías de la información y la comunicación}

Para lograr el desarrollo de la competencia comunicativa (y con la intención de innovar en el campo educativo teniendo en cuenta los gustos e intereses de los jóvenes en la actualidad), se pretende abrir un espacio a herramientas tecnológicas versátiles que favorezcan los procesos cognitivos. Aunque no existe una definición precisa de las TIC, las nuevas 
tecnologías de la información y la comunicación son aquellas que funcionan en tres ambientes básicos: la informática, la microelectrónica y las telecomunicaciones, las que a su vez se relacionan de manera interactiva e interconectada creando nuevas realidades comunicativas (Cabero, 1998). En consecuencia, el contexto virtual que se crea por medio del uso de estas tecnologías, es apropiado e innovador para generar y aplicar estrategias que redundan en el fomento de competencias comunicativas $\mathrm{y}$, a su vez, en la calidad educativa.

Dentro deestas tecnologías, encontramos diferentes dispositivos móviles como el celular y la Tableta, software educativo en línea y en tiempo real, sistemas digitales como las computadoras $\mathrm{u}$ ordenadores, sistemas de audiovisión como el televisor normal o el digital, videos y la Internet. Tales herramientas ofrecen opciones ilimitadas aplicables a cualquier campo de la actividad humana, desde lo cotidiano hasta las ciencias aplicadas. Es evidente que algunos de estos instrumentos son más utilizados que otros en el desarrollo de investigaciones, por su facilidad de adquisición, tendencia, innovación y constante evolución.

El dispositivo móvil más accesible y de mayor aceptación en la actualidad, es el teléfono móvil o celular, que cuenta con la mayoría de características ya mencionadas para darle relevancia sobre los demás, y ser usado como herramienta mediadora en el proceso de esta investigación. Un dispositivo móvil se puede definir como un aparato de pequeño tamaño, con algunas capacidades de procesamiento, con conexión permanente o intermitente a una red, con memoria limitada, que ha sido diseñado específicamente para una función, pero que puede llevar a cabo otras más generales.

Los docentes y los padres de familia consideran frecuentemente que el celular es más un obstáculo que una oportunidad para el aprendizaje. No obstante, en vez de recurrir a la común actitud represora, se puede llegar a utilizarlo como una experiencia educativa, ya sea para reflexionar acerca de su manejo responsable, o para utilizarlo como una herramienta más dentro del aula.

No se debe pasar por alto que los estudiantes tienen a su disposición un dispositivo multimedia, con cámara de fotos, cámara de video, grabadora de sonido con reproductor de audio, además de conexión inalámbrica a Internet. Todo esto hace del celular una herramienta privilegiada y a la mano de cualquier individuo. Su uso en el salón de clase, permite unir la cultura y los intereses de los estudiantes al proceso de aprendizaje, ayudando a la adquisición de conocimientos y motivando la interrelación docente-estudiante, la cual ha 'mutado' -si se permite el términoporque el desarrollo de la clase pasó de la «magistralidad» a la «colaboratividad», y el docente ha 'modificado' y adaptado su rol a las exigencias de la modernidad, actuando hoy como un guía e intercesor de los procesos de acceso a la información y el conocimiento en el aula.

Recientemente, la cantidad de fabricantes que incluyen excelentes
Los docentes y los padres de familia consideran frecuentemente que el celular es más un obstáculo que una oportunidad para el aprendizaje. 
La reflexión del proceso investigativo que se viene generando, se ajusta al paradigma cualitativo, debido a que es un fenómeno decididamente humanista para comprender la realidad social, percibida como la creatividad compartida de los individuos (Pérez, 1994). aplicaciones educativas para estos dispositivos, ha aumentado. Además, los usos que posee el celular van desde: registro de fotografías, videos, grabaciones de voz, intercambio de información, videoconferencias, chats, visualización de libros, documentos y material multimedia, manejo de aplicaciones; convirtiéndolo en una excelente herramienta para transformar la clase en una experiencia de aprendizaje cooperativo.

Además, existen razones a favor del uso de los dispositivos móviles en la escuela; por un lado, el uso del celular hace que los alumnos empleen la tecnología cotidiana en el contexto escolar; por el otro, si se utilizan estos dispositivos como herramienta para la construcción de conocimiento o recolección de datos en las actividades o la comunicación colaborativa, ayuda a los estudiantes a ser más competitivos en el mundo digital (Kolb, 2008).

No se puede negar que el uso de este dispositivo trae consigo también aspectos negativos, producto de su inadecuado manejo u orientación: la adicción o dependencia, convertirse en un distractor, acceso a información sin restricción, reductor de sociabilidad y bajo rendimiento escolar, de los cuales hemos sido testigos en la práctica diaria.

Es necesario caracterizar o tener en cuenta los rasgos y comportamientos actuales de los adolescentes para poder entender sus formas de pensar o actuar, así como cada uno de los factores que influyen en esos comportamientos. Se pretende conocerlos y manejar sus ritmos de aprendizaje con el fin de adaptar los métodos de enseñanza a esta nueva era tecnológica.

Esto podría llevar a concluir que los anteriores planteamientos sobre el adolescente, definen -de manera adecuada- algunas formas de promover el proceso de aprendizaje y la enseñanza a los estudiantes que atraviesan por esta etapa. También, deben dar la pauta a los docentes para organizar los contenidos, estrategias, y demás decisiones que son usadas en la práctica educativa diaria.

\section{Puntos de partida y llegada alrededor del proceso investigativo}

La reflexión del proceso investigativo que se viene generando, se ajusta al paradigma cualitativo, debido a que es un fenómeno decididamente humanista para comprender la realidad social, percibida como la creatividad compartida de los individuos (Pérez, 1994). Tiene un enfoque críticosocial, puesto que busca generar un cambio de pensamiento en el contexto educativo partiendo de las necesidades encontradas, ya que las ciencias sociales críticas no se contentan con producir saber nomológico, sino que se esfuerza por examinar la invariabilidad social, captando relaciones de dependencia susceptibles a cambio (McArthy, 1987). El trabajo se enmarca en el tipo Investigación Acción Educativa, puesto que interpreta lo que ocurre desde el punto de vista de quienes actúan e interactúan en la situación problema, es decir, que se debe aplicar, reflexionar y evaluar cada una de las actividades planteadas, sin descuidar el contexto (Elliott, 2005). 
La propuesta -que giró alrededor del dispositivo móvil, y que buscaba motivar e innovar- se conformó de varios elementos, a través de los cuales se esperaba mejorar los procesos en los educandos a corto o largo plazo; donde ellos reconocieron su autonomía, confianza, responsabilidad, motivación, gustos, espontaneidad, entre otros. Estas herramientas revelaron el perfil digital de los jóvenes de esta época, y se aprovecharon para mejorar una competencia que ha sido relegada: la oral, a la que no se le puede arrebatar la importancia que posee, siendo el medio más relevante de transferencia de información o contacto personal.

Desde la prueba titulada "Abre el baúl de tus recuerdos”, diseñada con el objetivo de realizar un diagnóstico sobre la competencia comunicativa oral expositiva de los estudiantes identificando los puntos de partida, para luego adaptar los talleres a las necesidades detectadas; se determinó cuáles fueron los aspectos que merecían mayor atención y práctica.

La prueba contenía nueve preguntas sobre información personal de los estudiantes y una serie de indicaciones para su realización. Tras la ejecución de este taller, se obtuvieron 41 videos filmados con los teléfonos móviles y pertenecientes a cada uno de los estudiantes, los cuales fueron evaluados todos con la rúbrica (ver tabla 1) que arrojó los niveles de desempeño en las categorías de paralenguaje, kinestesia, dominio del tema, organización, vocabulario y autodominio con una tendencia marcada en los niveles regular y por mejorar en casi todos los ítems evaluados, confirmando la necesidad de desarrollar una estrategia que mejore las debilidades en cuanto a la competencia comunicativa oral identificada en los estudiantes.

Hay que destacar que el uso del dispositivo móvil fue, quizás, la categoría que más resultados positivos arrojó en la etapa diagnóstica. Antes que nada, se realizó una serie de acuerdos para el manejo adecuado del dispositivo durante el trascurso de la aplicación de la estrategia, como: utilizarlo en el momento solicitado, buen manejo de los registros de video y audio obtenidos para que no se generara irrespeto, no desviar el objetivo de las actividades, entre otros.

Desde el momento en que se solicitó a los estudiantes la información sobre si contaban con un teléfono móvil para el desarrollo de las actividades, aumentó su motivación y la curiosidad sobre lo que se iba a realizar. Esta motivación es una de las categorías emergentes encontradas en la investigación, debido a la importancia de llevar a cabo los objetivos. Generó entusiasmo y acciones de progreso en el desarrollo de las diversas tareas, ya que se evidenció la seguridad al poder usar el teléfono móvil con libertad, debido a que, por reglamento institucional, está prohibido su uso en el aula de clase.

De la mano de esta, surge también la innovación en el aula, como otra categoría emergente. Aunque los jóvenes son nativos digitales, son expertos en el uso del teléfono móvil y pueden aportar muchas ideas o en ocasiones enseñar a un docente a usarlo; utilizar el celular
La propuesta -que giró alrededor del dispositivo móvil, y que buscaba motivar e innovar- se conformó de varios elementos, a través de los cuales se esperaba mejorar los procesos en los educandos a corto o largo plazo 
Tabla 1. Rúbrica de evaluación.

\begin{tabular}{|c|c|c|c|c|}
\hline \multicolumn{5}{|c|}{ RÚBRICA DE EVALUACIÓN 1} \\
\hline \multicolumn{5}{|c|}{ FECHA RECOPILACIÓN EVIDENCIA: } \\
\hline \multicolumn{5}{|c|}{ NOMBRE DEL ESTUDIANTE EVALUADO: } \\
\hline ÍTEM EVALUADO & \begin{tabular}{|c|} 
MUY BIEN \\
10 \\
\end{tabular} & $\begin{array}{c}\text { BIEN } \\
7 \\
\end{array}$ & $\begin{array}{c}\text { REGULAR } \\
5 \\
\end{array}$ & $\begin{array}{c}\text { POR MEJORAR } \\
2 \\
\end{array}$ \\
\hline \multirow{6}{*}{$\begin{array}{l}\text { PARALENGUAJE } \\
\text { Incluye todos los aspectos } \\
\text { que tiene que ver con el uso } \\
\text { de la voz como la dicción, } \\
\text { tono, pausas, ritmo y } \\
\text { entonación. }\end{array}$} & \multirow{6}{*}{$\begin{array}{l}\text { Utilizó } \\
\text { adecuadamente } \\
\text { todos los elementos } \\
\text { del paralenguaje: } \\
\text { dicción, tono, } \\
\text { pausas, ritmo y } \\
\text { entonación. }\end{array}$} & $\begin{array}{l}\text { Utilizó adecuadamente } \\
\text { al menos cuatro } \\
\text { elementos del } \\
\text { paralenguaje. }\end{array}$ & \begin{tabular}{|l} 
Utilizó \\
adecuadamente al \\
menos tres elementos \\
del paralenguaje. \\
\end{tabular} & \begin{tabular}{|l} 
Utilizó \\
adecuadamente \\
menos de tres \\
elementos del \\
paralenguaje. \\
\end{tabular} \\
\hline & & Dicción & Dicción & Dicción \\
\hline & & Tono & Tono & Tono \\
\hline & & Pausas & Pausas & \\
\hline & & Ritmo & Ritmo & \\
\hline & & Entonación & Entonación & Entonación \\
\hline $\begin{array}{l}\text { ORGANIZACIÓN } \\
\text { Es la forma como } \\
\text { estructura el mensaje } \\
\text { que se quiere comunicar, } \\
\text { desarrollando una } \\
\text { secuencia lógica: } \\
\text { introducción, desarrollo y } \\
\text { conclusión. }\end{array}$ & $\begin{array}{l}\text { Cumplió con la } \\
\text { estructura precisa } \\
\text { de un discurso } \\
\text { expositivo. }\end{array}$ & $\begin{array}{l}\text { Cumplió con la } \\
\text { estructura de un } \\
\text { discurso expositivo, } \\
\text { pero presenta algún } \\
\text { error. }\end{array}$ & $\begin{array}{l}\text { La estructura presentó } \\
\text { inconsistencias que le } \\
\text { quitaron objetividad } \\
\text { al discurso expositivo. }\end{array}$ & $\begin{array}{l}\text { Incumplió con la } \\
\text { estructura de un } \\
\text { discurso expositivo; } \\
\text { faltó objetivo, } \\
\text { presentó poca } \\
\text { información. }\end{array}$ \\
\hline $\begin{array}{l}\text { DOMINIO DEL TEMA } \\
\text { Habla con propiedad y } \\
\text { efectividad sobre el tema } \\
\text { que se le pregunta. }\end{array}$ & $\begin{array}{l}\text { Evidenció tener } \\
\text { conocimiento del } \\
\text { tema. }\end{array}$ & $\begin{array}{l}\text { Tiene conocimiento } \\
\text { del tema con algunas } \\
\text { dudas. }\end{array}$ & $\begin{array}{l}\text { Presentó inseguridad } \\
\text { sobre lo que exponía } \\
\text { y omitió información. }\end{array}$ & $\begin{array}{l}\text { Mostró evidente } \\
\text { desconocimiento del } \\
\text { tema. }\end{array}$ \\
\hline $\begin{array}{l}\text { VOCABULARIO } \\
\text { Manejo de palabras } \\
\text { acordes, adecuadas y } \\
\text { variadas a la información } \\
\text { que debe dar a conocer. }\end{array}$ & $\begin{array}{l}\text { Se utilizó un } \\
\text { vocabulario } \\
\text { adecuado y variado. }\end{array}$ & $\begin{array}{l}\text { Su vocabulario es } \\
\text { adecuado y variado } \\
\text { con pocos errores. }\end{array}$ & $\begin{array}{l}\text { Tuvo inconsistencias } \\
\text { en el uso del lenguaje } \\
\text { y utilizó algunas } \\
\text { muletillas. }\end{array}$ & $\begin{array}{l}\text { Se utilizó un } \\
\text { vocabulario pobre } \\
\text { y deficiente con uso } \\
\text { de muletillas. }\end{array}$ \\
\hline \multirow{5}{*}{$\begin{array}{l}\text { KINESTESIA } \\
\text { Todo lo que tiene que } \\
\text { ver el contacto visual, la } \\
\text { postura, expresión facial y } \\
\text { ademanes. }\end{array}$} & \multirow{5}{*}{$\begin{array}{l}\text { Utilizó } \\
\text { adecuadamente } \\
\text { todos los elementos } \\
\text { de la kinestesia: } \\
\text { contacto visual, la } \\
\text { postura, expresión } \\
\text { facial y ademanes. }\end{array}$} & $\begin{array}{l}\text { Utilizó adecuadamente } \\
\text { tres elementos de la } \\
\text { kinestesia. }\end{array}$ & $\begin{array}{l}\text { Utilizó } \\
\text { adecuadamente } \\
\text { dos elementos de la } \\
\text { kinestesia. } \\
\end{array}$ & $\begin{array}{l}\text { Utilizó } \\
\text { adecuadamente } \\
\text { un elemento de la } \\
\text { kinestesia. } \\
\end{array}$ \\
\hline & & Contacto visual & Contacto visual & \begin{tabular}{|l|l} 
Contacto visual \\
\end{tabular} \\
\hline & & Postura & Postura & Postura \\
\hline & & Expresión facial & Expresión facial & Expresión facial \\
\hline & & Ademanes & Ademanes & Ademanes \\
\hline $\begin{array}{l}\text { AUTODOMINIO } \\
\text { Capacidad de controlar los } \\
\text { impulsos y los nervios. }\end{array}$ & $\begin{array}{l}\text { Controló } \\
\text { adecuadamente el } \\
\text { nerviosismo. }\end{array}$ & $\begin{array}{l}\text { Controló } \\
\text { adecuadamente el } \\
\text { nerviosismo con } \\
\text { alguna vacilación. }\end{array}$ & $\begin{array}{l}\text { Tuvo inconsistencia } \\
\text { en el control del } \\
\text { nerviosismo. }\end{array}$ & $\begin{array}{l}\text { Carencia de control } \\
\text { de nervios. }\end{array}$ \\
\hline
\end{tabular}

Fuente: tomado y adaptado de: Estrategias de Enseñanza para el Desarrollo de la Competencia Comunicativa Oral. (Hernández, 2012, p. 123). 
como una herramienta pedagógica, no es su fuerte. Por tal razón, esta oportunidad cambió la rutina académica en la que se desenvuelven de manera tediosa y desobligante, a una dinámica y liberadora que se evidenció por su participación activa, interés por realizar nuevas propuestas para los ejercicios, entusiasmo y el solicitar la oportunidad o más tiempo para hacerlo mejor.

En último lugar, emerge el trabajo colaborativo en la interacción de los integrantes de los equipos de trabajo al construir juntos, conjugar esfuerzos, habilidades, talentos y competencias que les permitieron lograr las metas establecidas (Maldonado, 2007). La cooperación surgió aprendiendo el uno del otro, intercambiando conocimiento acerca del manejo del dispositivo móvil o de las sugerencias para las respuestas o desempeño en la prueba.

Estas subcategorías emergentes consolidan y justifican la importancia del dispositivo móvil como herramienta pedagógica, y conforman el conjunto de elementos necesarios para la consecución del objetivo planteado, implicando crear un cambio necesario en los roles que el estudiante y el docente tienen en la enseñanza tradicional; de manera que, el aprendizaje debe ser tomado como una actividad en la que le estudiante indaga, valora, emplea, incorpora y viaja por la información disponible.

De acuerdo con los resultados arrojados en la prueba diagnóstica, se diseñó una estrategia de mejoramiento de los elementos que conforman la competencia oral expositiva, compuesta por tres talleres y usando el teléfono móvil como herramienta para el desarrollo de las actividades. Se diseñaron 3 instrumentos con diferentes actividades donde el celular cumplió diversas funciones, y cada uno tenía un objetivo distinto apuntando a cumplir con la meta de la investigación.

El taller 1, "Soltemos la lengua", se centró en la realización de ejercicios para desarrollar o perfeccionar los aspectos que tienen que ver con el paralenguaje: dicción, tono, pausas y entonación, en el cual se solicitó la creación de audios con las cinco tareas a realizar y ser enviados a las docentes encargadas vía bluetooth o WhatsApp. Se encontró que los estudiantes no mostraban naturalidad en sus expresiones y, con gran dificultad, debían esforzarse por decir las frases con la entonación adecuada, rasgos de nerviosismo, mala articulación de los sonidos al hablar, uso inadecuado de las pausas y poca fluidez.

En cuanto al uso del celular, en este taller también se notó mayor motivación porque se redujo la ansiedad, debido a que el producto a entregar era un audio y los estudiantes no se sintieron intimidados por la cámara; siendo más natural su desempeño. Además, ampliaron sus conocimientos o experiencias al buscar diferentes formas de crear y enviar los audios, fueron muy recursivos, los que no tenían la aplicación de grabación de voz los hicieron creando los audios directamente en WhatsApp.

Por lo que respecta al taller 2, titulado "Palabras correctas", este buscó fomentar la habilidad para manejar un vocabulario más amplio y acorde a temáticas específicas de un área. El taller
En último lugar, emerge el trabajo colaborativo en la interacción de los integrantes de los equipos de trabajo al construir juntos, conjugar esfuerzos, habilidades, talentos y competencias que les permitieron lograr las metas establecidas (Maldonado, 2007). 
Cabe anotar que, estos avances en las habilidades orales son mínimos comparados con el trabajo que se debe realizar desde todos los ámbitos, no solo de la escuela, sino en la vida diaria. consistió en el manejo en grupo de una aplicación móvil, la creación de una narración con la participación de todos los estudiantes, y un juego de palabras y velocidad, relacionadas con un tema determinado.

Por medio del manejo de la aplicación y la realización de los ejercicios, se percibió un mejor desempeño, puesto que se utilizó un vocabulario más pertinente y hubo mayor participación de los estudiantes. Todo lo anterior lleva a afirmar que el vocabulario, el autodominio y el dominio del tema, están encadenados y la presencia de cada uno hace posible la existencia de los otros, como elementos esenciales de la competencia comunicativa oral expositiva.

Por último, el taller 3, "Sin miedo a hablar", se ocupó de fomentar y mejorar aspectos relacionados con la kinestesia, organización y dominio del tema, obviamente sin dejar de lado los anteriormente analizados. Para esto, se desarrolló un taller de entrenamiento que consistía en el manejo de la aplicación "La oratoria", síntesis de recomendaciones sobre la expresión oral, trabajo en equipo y creación de un video tutorial en grupo en donde todos los integrantes explicasen de manera creativa, recursiva y dinámica, cómo realizar una exposición oral. Se hizo énfasis en la importancia del dominio que tenían que tener sobre el tema, la organización de las ideas y, más aún, la preparación del material, el entorno y su participación individual.

Después de evaluados los videos, se notó un gran avance en cuanto a la actitud se refiere, en los primeros talleres, aunque los estudiantes también estaban motivados, generaron cierto tipo de resistencia y el nerviosismo impedía su naturalidad, pero en general fueron más expresivos, pusieron en práctica la mayoría de elementos tratados en los anteriores talleres.

Cabe anotar que, estos avances en las habilidades orales son mínimos comparados con el trabajo que se debe realizar desde todos los ámbitos, no solo de la escuela, sino en la vida diaria. La competencia comunicativa debe ser un eje transversal en todas las áreas, aunque no cuenta con criterios de evaluación y es un aspecto secundario que depende de cada docente; es de suma importancia reivindicar su lugar sabiendo que el ser humano destina la mayoría de su tiempo a la comunicación y, en gran porcentaje, a hablar.

La etapa final de la investigación fue una prueba titulada "Llegó la hora: exprésate correctamente", que consistió en preparar una exposición individual sobre un tema determinado y entregado con varios días de anticipación a la presentación para su preparación; se debía tener en cuenta cada uno de los consejos dados en los talleres, las habilidades practicadas y la experiencia adquirida.

Los resultados indicaron, en primer lugar, el cambio que presentaron los estudiantes frente al componente del paralenguaje, donde se preocuparon por expresar con mayor claridad sus ideas, pronunciando más lenta y correctamente. Así mismo, se interesaron por dar cumplimiento a la prueba haciendo uso 
de lo aprendido en los talleres, fijándose en su ritmo en el habla, las pausas adecuadas para no cambiar la intención del mensaje. Persistió el tono de voz bajo, tal vez, consecuencia de los nervios al ser filmados, y tener un público más amplio y atento a la presentación.

En segundo lugar, se percibió mayor compromiso en cuanto a la organización del tema propuesto, estructurando las ideas de manera lógica y coherente -evidenciando preparación e investigación- cumpliendo con los parámetros establecidos para el desarrollo de la exposición, como el tiempo, las ayudas visuales y su presentación. Lo anterior contribuyó a aumentar la seguridad al hablar, pues conocían el tema y expresaron mensajes claros y precisos, aspectos necesarios para demostrar dominio del tema.

De modo similar, el uso del vocabulario fue más pertinente y lógico, con pocas muletillas, teniendo relación con el tema expuesto, debido a la apropiación del mismo, y como resultado se apreció un enriquecimiento en el saber de los participantes. El empleo de nuevo vocabulario incrementa su léxico cotidiano, al comprender el significado de nuevos vocablos y nutre, a su vez, otras habilidades como la escritura.

Como es sabido, el ser humano no solo se comunica por medio de lo que dice, sino también por la forma en que lo dice, hecho que se evalúa con la kinestesia. El progreso de este aspecto se reflejó notablemente en las buenas posturas adoptadas por los estudiantes, mayor contacto visual al público y cámara del dispositivo móvil, sin gestos exagerados ni risa nerviosa; controlando también los movimientos constantes de manos y pies, que demostraron en la prueba diagnóstica. Razón por la cual, el autodominio se intensificó al controlar las emociones, siendo capaces de expresar sus ideas con mayor seguridad y poco nerviosismo.

Como punto final, siendo la meta de esta investigación descubrir cómo el uso del celular mejora la competencia comunicativa oral expositiva (sabiendo $\mathrm{y}$ aprovechando que los estudiantes están en una era digital), se puede afirmar que la inclusión de un dispositivo tecnológico en el proceso educativo es clave para mejorar los métodos de enseñanza, el aprendizaje autónomo y colaborativo que, a largo plazo, potenciarán la calidad educativa.

Con el aumento de la motivación generado desde el comienzo de la aplicación de la estrategia, se fue incrementando la autonomía en los estudiantes en la toma de decisiones, su participación activa, su interés por hacer las actividades de manera adecuada sin falta de casos puntuales de apatía, negativismo o poco compromiso- $\mathrm{y}$ querer siempre colaborar con sus compañeros que no contaban con un dispositivo móvil o con las habilidades necesarias para su manejo.

Por lo tanto, el uso del teléfono móvil generó un impacto positivo en el cumplimiento del objetivo de la investigación al crear innovación involucrando en el aula los "juguetes multimedia" a las actividades educativas y así los estudiantes pueden aprender cómo usarlos como herramientas
Como punto final, siendo la meta de esta investigación descubrir cómo el uso del celular mejora la competencia comunicativa oral expositiva (sabiendo y aprovechando que los estudiantes están en una era digital), 


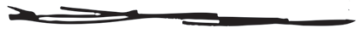

El docente es responsable de escoger los instrumentos necesarios y eficaces para poder evaluar las destrezas comunicativas. de conocimiento (Kolb, 2008, p. 7), dejando atrás la censura existente por el empleo de esta herramienta en los establecimientos educativos.

El docente es responsable de escoger los instrumentos necesarios y eficaces para poder evaluar las destrezas comunicativas. A largo plazo, se pretende implementar en el currículo de la institución esta estrategia -como un eje transversal en todas las áreas del conocimiento- debido a la importancia que adquiere no solo el hablar correctamente, sino crear identidad y fortalecer la personalidad de los educandos.

Así mismo, los estudiantes desarrollan estrategias necesarias para generar procesos comprensivos que mejoran la competencia lingüística y comunicativa, fortaleciendo la cultura digital. No solo en ellos, sino también en los docentes que hacen uso de las herramientas de información y comunicación, creando un nuevo conocimiento a través de una alternativa innovadora en el proceso enseñanza-aprendizaje acorde a la dinámica global actual.

Al aplicar la estrategia pedagógica usando el teléfono móvil como herramienta didáctica, se percibió gran motivación en los estudiantes debido a la innovación que generaba el uso de este dispositivo prohibido en las demás clases. Este hecho contribuyó, de manera sustancial, en el óptimo desarrollo de los talleres diseñados para perfeccionar las habilidades de la competencia comunicativa oral expositiva. Todo esto fue posible a través de la multifuncionalidad que posee el móvil, ofreciendo ubicuidad para ser usado en cualquier momento y lugar, portabilidad por su pequeño tamaño, interactividad para que el estudiante sea partícipe de su propio saber y colaborativo permitiendo el aprendizaje por pares.

Por consiguiente, se generó un ambiente de aprendizaje adecuado a los intereses de los jóvenes participantes de la investigación, ya que se propiciaron espacios de exploración en donde ellos debían fusionar sus ideas, imaginación y motivación para dar origen a diferentes productos que permitieron evaluar el progreso y desempeño en las actividades de los talleres. Así mismo, este entorno fue muy favorable para perfeccionar algunas de las habilidades comunicativas de los estudiantes por medio de los ejercicios planteados.

Finalmente, se podría afirmar que el desarrollo de la competencia comunicativa oral y el manejo adecuado del teléfono móvil como herramienta educativa, son acciones continuas que deben seguir perfeccionándose en el tiempo, el contexto y la necesidad que la diaria labor docente lo demande. 


\section{Referencias}

Abascal, M. (2002). La teoría de la oralidad. Alicante, España.

Cabero, J. (1998). Impacto de las nuevas tecnologías de la información y la comunicación en las organizaciones educativas. En M. Lorenzo et al. (coords), Enfoques en la organización y dirección de instituciones educativasformales y no formales (pp. 197-206). Granada: Grupo Editorial Universitario.

Cassany, D., \& Luna, M. (1998). Enseñar lengua. Barcelona: Graó.

ElLiotT, J. (2005). El cambio educativo desde la investigación acción. Madrid: Ediciones Morata.

HERNÁNDEZ, L. (2012). Estrategias de enseñanza para el desarrollo de la competencia comunicativa oral en los alumnos que cursan lengua española. México: Tecnológico de Monterrey.

Hymes, D. (1996). Acerca de la competencia comunicativa (J. Gómez Bernal, Trad.). Forma y función, (9), 13-37.

Jáuregr, K., \& SANZ (2006). Mis estudiantes son diginativos iy los tuyos? El aprendizaje de la competencia comunicativa oral a través de entornos digitales: un reto posible. Recuperado de https://cvc.cervantes.es/ensenanza/biblioteca_ele/asele/ pdf/17/17_1163.pdf

KolB, L. (2008). Toys to tools: Connecting Student Cell Phones to Education. Washington D. C.: International Society for Technology in Education.

Maldonado, M. (2007). El trabajo colaborativo en el aula universitaria. Revista de educación Laurus, 13(23), 263-278.

MARTínez, C. (2012). El desarrollo de la competencia oral en la enseñanza aprendizaje del español como lengua extranjera a través del uso de las tecnologías de la información y comunicación. (Tesis doctoral). Universidad de Granada, Granada, España. Recuperado de http://digibug.ugr.es/bitstream/10481/24554/1/21613412.pdf

McArthy, T. (1987). La teoría Crítica de Jürgen Habermas. Madrid: Tecnos.

Monsalve, M. E. (2009). Desarrollo de las habilidades comunicativas en la escuela nueva. Educación y pedagogía, 21(55), 189-210.

NARVÁEZ, J. (2014). Estrategias para motivar el desarrollo de la expresión oral en los estudiantes de inglés bajo intermedio del curso 10-3 del instituto Champagnat de pasto, año 2012. Universidad de La Sabana, Bogotá, Colombia.

NuÑEz, M. (2004). La competencia discursiva oral y la interacción en el aula del EL2. Ceuta, España: Universidad de Granada.

OspinA, S. (2011). El desarrollo de la metacognición y de la competencia estratégica oral mediante el uso de internet. En C. Hernández, A. Carrasco y E. Álvarez (eds.), La Red y sus aplicaciones en la enseñanza-aprendizaje del español como lengua extranjera (pp. 365-375). Valladolid: ASELE.

PÉREZ, G. (1994). Investigación educativa: Retos e interrogantes. Madrid: La Muralla S.A.

Ramos, A. H. (2010). Desarrollo de habilidades cognitivas con aprendizaje móvil: un estudio de casos. Revista Científica de Educomunicación, 17(34), 201-209.

Ramos, A., \& Caurcel, M. (2011). Los podcasts como herramienta de enseñanza aprendizaje en la universidad. Profesorado. Revista de Currículum y Formación de Profesorado, 15(1), 151-162. 
Rosales, B., Zarate, J., \& Lozano, A. (2013). Desarrollo de la competencia comunicativa en el idioma inglés en una plataforma interactiva. Revista electrónica Sinéctica, (41), 1-11. 